\title{
Perspective
}

PERSPECTIVE Actualité en histoire de l'art

$2 \mid 2020$

Danser

\section{« En verve »- Illustrer le livre de cuisine : photographie culinaire et imaginaire pictural}

"En verve" - Illustrating the Cookbook: Culinary Photography and the Pictorial Imaginary

„In Form“. Über das Illustrieren des Kochbuchs: Food-Fotografie und bildliche Vorstellung

"En verve" - Illustrare il libro di cucina: fotografia culinaria e immaginario pittorico

"Con labia" - Ilustrar el libro de cocina: fotografía culinaria e imaginario pictórico

\section{Guillaume Cassegrain}

\section{(2) OpenEdition}

Journals

Édition électronique

URL : https://journals.openedition.org/perspective/21702

DOI : 10.4000/perspective.21702

ISSN : 2269-7721

Éditeur

Institut national d'histoire de l'art

\section{Édition imprimée}

Date de publication : 30 décembre 2020

Pagination : 251-260

ISBN : 978-2-917902-90-5

ISSN : $1777-7852$

Référence électronique

Guillaume Cassegrain, « «En verve » - Illustrer le livre de cuisine : photographie culinaire et imaginaire pictural », Perspective [En ligne], 2 | 2020, mis en ligne le 30 juin 2021, consulté le 30 juillet 2022. URL http://journals.openedition.org/perspective/21702; DOI : https://doi.org/10.4000/perspective.21702 


\title{
« En verve»
}

\section{Illustrer le livre de cuisine : photographie culinaire et imaginaire pictural}

\author{
Guillaume Cassegrain
}

- Servez citron..., 2020 : Servez citron. Un ensemble de photographies par Éric Poitevin

d'assiettes desservies chez Troisgros, accompagné des recettes afférentes [par Michel

et César Troisgros], piqué de "Restes de table ", un essai par Jean-Claude Lebensztejn,

Paris, Macula, 2020.

L'enfermement imposé par les gouvernements du monde en réponse à la circulation trop active du virus SRAS-CoV-2, probablement propagé chez l'homme par la consommation de viande de civette ou de pangolin infectée ${ }^{1}$, a eu pour effet, notamment, de donner ou de redonner goût à la cuisine, et de manger un peu plus qu'à l'habitude (entrainant une prise de poids moyenne de $2 \mathrm{~kg}$ par habitant). Cet engouement s'est accompagné du développement d'une mode, déjà présente auparavant chez ceux que l'on appelle les foodies (version moderne des "gourmets " des années passées ou de ceux, encore plus anciens, qui maitrisaient la " science de gueule $\left.{ }^{2} »\right)$, consistant à prendre en photo (la plupart du temps avec son smartphone) l'assiette que l'on aura préparée ou, lorsque l'on est au restaurant, le plat que l'on vient de servir. Les amateurs de cuisine comme les cuisiniers amateurs ont ainsi échangé, depuis leur lieu de réclusion, des photographies de leurs dernières créations, inondant la toile d'images de plats plus ou moins alléchants.

Si les chefs (certains d'entre eux, tout au moins) ont accueilli avec bienveillance la conversion médiatique de la cuisine (grande ou plus petite) et profité de ces multiples expositions audiovisuelles (livres, émissions de télévision, chaines YouTube, etc.), pour le meilleur mais aussi pour le pire, ils ont également été débordés par ce déferlement d'images sur les réseaux sociaux. De nombreux grands cuisiniers (que disent ces termes, comme " chefs ", visant une certaine catégorie de cuisiniers, les "grands ", les "étoilés ", par exemple, avec son vocabulaire militaire ? Face à ces " noms", on trouve aussi le bataillon plus anonyme des " tantes ", " sœurs ", " grand-mères " ") se sont opposés à cette prolifération des photographies de plats en interdisant toute prise d'image dans leurs restaurants, afin de défendre la signature de leurs créations culinaires (il n'existe pas de moyens juridiques de protéger une recette comme une propriété intellectuelle si ce n'est, peut-être, en la publiant et en la photographiant, car l'image et le texte sont reconnus comme des œuvres relevant du droit d'auteur ${ }^{4}$ ) mais aussi de conserver une part de la surprise visuelle espérée au moment du service (la cloche, passée de mode, remplissait aussi cette fonction d'aménagement d'une révélation visuelle et olfactive). 


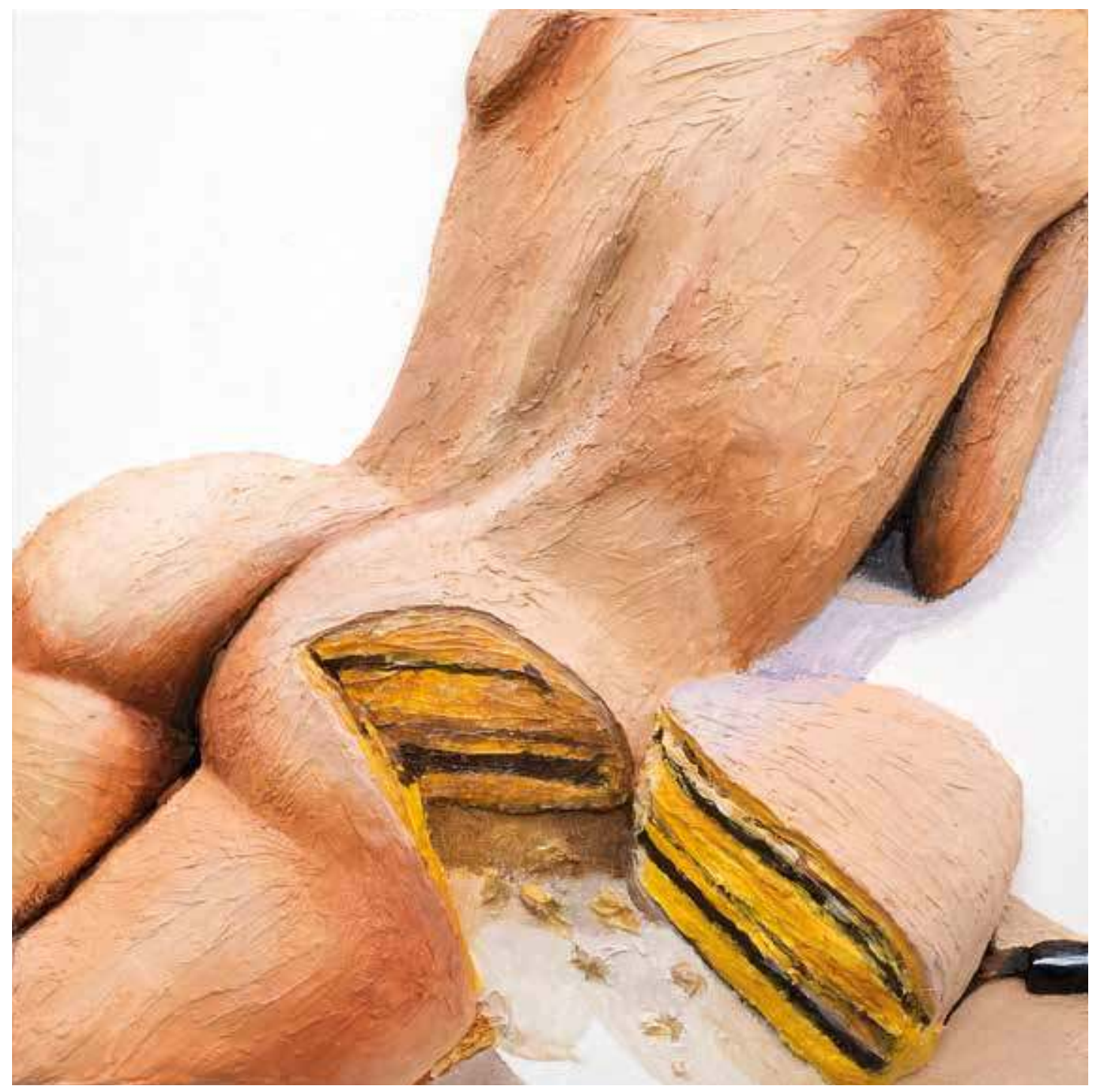

1. Gina Beavers, Cake, 2015, Ces millions de photographies échangées chaque jour sur les peinture acrylique sur toile. réseaux sociaux, depuis un restaurant huppé ou un kebab sans charme (l'inverse est également possible), ont favorisé l'apparition d'un genre nouveau qui a trouvé assez tôt le nom étrange de foodporn. Ce terme ne désigne pas (on pourrait s'y méprendre) ce qu'une tradition, relayée sous différents registres par l'art contemporain ${ }^{5}$ (fig. 1), peut faire avec la nourriture, détournée de sa destination première, dans le cadre d'activités sexuelles; comme dans les romans de Sade où les nourritures et les corps s'échangent, se complètent ou se substituent les uns aux autres : les victimes sexuelles devenant les tables où les aliments sont disposés et consommés ; les vins rares servant à se laver " les fesses, le cou, les aisselles, la bouche et le trou du cul ${ }^{6}$ ". Et encore, la femme aimée de Stéphane Mallarmé, Méry Laurent, qui est, tour à tour, une " dame crémeuse ", une " chère meringue ${ }^{7}$ ". Roland Barthes, dans sa préface à la Physiologie du goût de Jean Anthelme Brillat-Savarin, avait déjà pointé ce rapport entre 
perversion sexuelle et perversion gastronomique et trouvé, entre ces deux registres, une même appétence pour une érotique du regard : " Il rôde autour de la table une vague pulsion scopique : on regarde (on guette?) sur l'autre les effets de la nourriture, on saisit comment le corps se travaille de l'intérieur; tels ces sadiques qui jouissent de la montée d'un émoi sur le visage de leur partenaire, on observe les changements du corps qui se nourrit bien ${ }^{8}$."

Originairement destiné à assumer pleinement des désirs de nourriture - grasse, sucrée, abondante et pas nécessairement raffinée (des hamburgers dégoulinant de fromage fondu agrémenté de coquillettes, par exemple), puis élargi à cette habitude d'exalter le plat que l'on mange en l'immortalisant par une photographie, le terme de foodporn a été forgé à partir de gastro-porn, inventé à l'occasion de la recension d'un livre de recettes de Paul Bocuse dans lequel les illustrations des plats, si suggestives, du chef lyonnais faisaient savourer les lecteurs par avance (fig. 2). Le gastro-porn que décrivit alors Alexander Cockburn ${ }^{9}$ à la lecture du French Cooking de Paul Bocuse, traduction (mais que traduit-on dans un livre de cuisine ? Les aliments ou condiments pouvant vite devenir intraduisibles d'un pays à l'autre, comme l'agretto, vinaigre fait à partir des raisins servant au vino santo, qui sera " traduit " par un vin blanc d'Orléans ${ }^{10}$. Carl Friedrich von Rumohr pensait de même que la piètre qualité gustative d'un plat " archi-épicé et aussi sec qu'une momie " pouvait venir d'une mauvaise traduction de la recette latine originale ${ }^{11}$ ) de sa Cuisine $d u$ marché. En hommage à Alfred Guérot (Paris, Flammarion, 1977), un best-seller du livre de cuisine, tire son plaisir non seulement de la lecture des recettes mais aussi de la qualité " esthétique " des reproductions des plats proposés. Cockburn relevait le rôle, de plus en plus important, que la photographie des plats tenait dans un livre de recettes, et des effets suggestifs qu'elle produisait lorsque le travail de composition, de cadrage, de coloration était bien fait. La photographie des plats, qu'elle soit le fait d'un photographe professionnel ${ }^{12}$, spécialisé dans ce genre et préoccupé de considérations avant tout techniques et pratiques (identification facile des aliments, présentation claire du plat, éclairage satisfaisant, etc.) ou d'un artiste reconnu dans le milieu de l'art contemporain, s'est vue placée soudainement sous la loi des évolutions stylistiques et des goûts changeants que les documents techniques ignoraient alors. Ainsi les photographies des plats de Bocuse, qui semblaient si alléchantes au lecteur de ces années 1970, sont-elles devenues, avec le temps, démodées et peu ragoûtantes, avec leur teinte marron, leur dispositif "petit bourgeois " (verre de vin, généralement rouge, posé à l'arrière-plan, parfois accompagné d'une bouteille, table en bois massif, napperon, etc.), et ces documents ont-ils basculé dans la vaste et indistincte histoire du style, imposant de procéder à de nouvelles prises de vue à chaque réédition.

Le gastro-porn célébrait le rôle de plus en plus important que l'illustration jouait dans l'intérêt porté aux livres de cuisine et magnifiait cette "pulsion scopique " dans le commerce avec la nourriture. Pratiques largement partagées, désormais, par les amateurs de cuisine, et qui auraient fait bondir d'horreur les maîtresses de maison habituées aux bonnes manières, chez qui la qualité du repas servi ne devait

2. Photographie illustrant la recette de la célèbre soupe à la truffe "Élysée » de Paul Bocuse, dans Paul Bocuse's French Cooking, New York, Pantheon Books, 1977, p. 59.

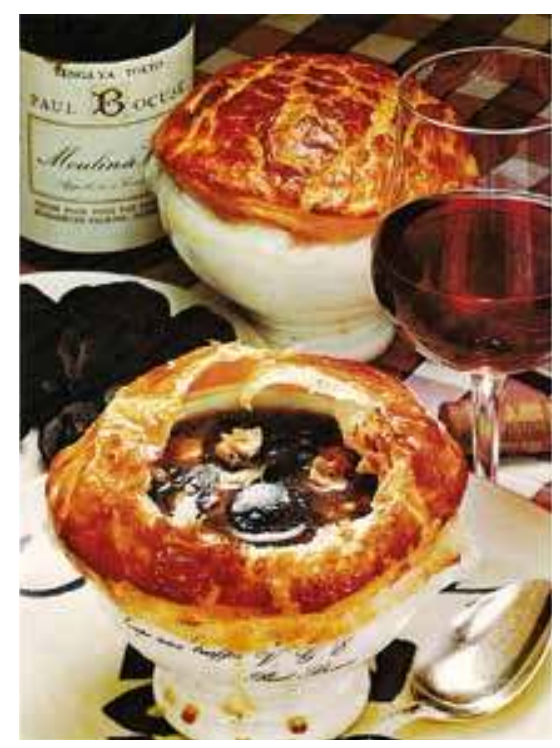


pas être abordée par les convives. Jean-Claude Lebensztejn, dans son essai introductif à l'ouvrage Servez citron..., repris des Manières de table publiées en 2004 et mises au pilon, " sans manière ", par l'éditeur, merveilleux hors-d'œuvre, rappelle ces règles : " Et vers 1700 saint Jean-Baptiste de La Salle, à la suite de Courtin, conseille à ses élèves de ne pas trop commenter ce qu'on mange, "car ce seroit faire paroistre qu' on prend bien du plaisir dans la bonne chere"13. " La comtesse de Boissieu, comme beaucoup d'autres, donne ce même conseil dans son manuel de savoir-vivre plus tardif : " "On ne doit pas vivre pour manger mais on doit manger pour vivre." De cet axiome résulte le devoir de ne pas ajouter une trop grande importance au fonds même du repas. Ainsi, on évitera de louer ou de critiquer les mets qui sont servis ; on s'abstiendra de porter ou de maintenir la conversation sur ce qui a rapport à la table ${ }^{14}$. "

Les auteurs de livres de cuisine comme, plus généralement, ceux qui entendent, par des conseils pratiques ${ }^{15}$ ( " changer l'eau de temps en temps ${ }^{16}$ ", " ne pas les filmer ${ }^{17}$ ", etc.), transmettre un savoir-faire, ont été très tôt confrontés au nécessaire accompagnement visuel du texte. Comme toutes pratiques manuelles, la cuisine n'a pu se contenter de simples descriptions, aussi précises soient-elles, pour expliquer, à distance, les étapes indispensables à la bonne réalisation d'un plat. Les recettes proposées par Michel et César Troisgros s'en tiennent aux modalités classiques de ce genre littéraire en recourant systématiquement, même en adoptant l'indicatif, au mandat impératif ( Peler les pommes ", " Éplucher les oignons ", " Déposer les cervelles "...) afin d'investir le destinataire, comme l'a si scrupuleusement montré Algirdas J. Greimas dans son célèbre texte sur la soupe au pistou ${ }^{18}$, d'un " savoir-faire " permettant l'accomplissement de ce " programme narratif " et la bonne performance du plat fini, grâce à la description qui est proposée et à la photographie qui fixe le modèle à reproduire.

La médecine (la chirurgie ${ }^{19}$, l'obstétrique, etc.), les sciences naturelles, tout ce savoir théorique qui dépend d'une pratique se sont aussi développés et ont été enseignés par l'association du texte et des images ${ }^{20}$. Les illustrations ont ainsi, très tôt, accompagné les livres de cuisine (qu'il s'agisse de rassembler des recettes ou des menus entiers, de décrire les ustensiles de cuisine ou le lieu lui-même), soit sous la forme de vignettes discrètes et ornementales, soit sous celle de planches destinées à soutenir plus efficacement le texte. Le célèbre livre de Bartolomeo Scappi, Premier traité de cuisine illustré, comptant 500 recettes accompagnées de 27 gravures dans son édition de 1570, réserve des planches entières à des illustrations servant d'accompagnement pédagogique au texte ${ }^{21}$. Des représentations détaillées de cuisines idéales, avec les multiples tables destinées aux différentes tâches de la préparation des plats (celle pour la découpe des viandes, celle pour le travail des pâtes...), les feux, les réserves, etc., voisinent avec la gravure d'instruments (couteaux, bassines...) nécessaires à la réalisation des recettes contenues dans l'ouvrage. Des représentations plus précises de tâches pratiques (la découpe des viandes, par exemple) viennent aider le lecteur dans sa volonté de reproduire ce que l'auteur lui enseigne. Cet accompagnement didactique où l'image allait jouer progressivement un rôle essentiel, s'adaptant, à l'occasion des lecteurs, par des variations stylistiques, comme dans le célèbre La cuisine est un jeu d'enfants de Michel Oliver ${ }^{22}$ où les dessins de la main de l'auteur imitent ceux des enfants, a contribué à transformer le contenu des livres de cuisine et à les rapprocher des livres d'art.

Les recettes pratiques n'ont plus suffi à contenter l'intérêt des amateurs de cuisine, et les éditeurs, dans un secteur fortement concurrentiel, ont dû faire preuve d'imagination pour que leurs productions se singularisent par rapport aux autres. Le livre de cuisine est devenu un " objet " dont les contours n'ont cessé d'évoluer au gré des modes, et dont l'aspect esthétique peut parfois devenir si déroutant que l'on ne sait s'il vaut pour sa prouesse 
visuelle ou pour ses conseils de cuisine. La dimension pratique de l'ouvrage, bien loin néanmoins de disparaître entièrement au profit de sa métamorphose en livre d'art, s'est développée en proposant notamment des couvertures lavables (l'auréole blanche qui semble tacher la couverture de Servez citron... pourrait être une allusion à ces questions d'usage), des reliures facilement pliables, des livres " chevalets", des livres " coffrets " dans lesquels le lecteur trouve des ustensiles nécessaires à la réalisation des recettes (rouleau à pâtisserie, siphon, moule... $)^{23}$. Le compagnonnage avec l'art a permis au livre de cuisine de valoriser, esthétiquement et commercialement, son sujet et d'accompagner ce " faire persuasif " qui est à l'œuvre dans les recettes proposées, part importante du « programme de l'auteur soucieux de faire vendre son livre de cui$\operatorname{sine}^{24} »$. Les allusions à l'art (la peinture, principalement) sont rapidement devenues innombrables dans cette littérature culinaire et se situent à des niveaux divers. Les recettes peuvent venir, directement ou plus indirectement, des artistes eux-mêmes, et inviter le lecteur à partager la " table de " Cézanne, Monet ou Renoir ${ }^{25}$ pour déguster les plats aimés ou imaginés par les artistes, mais elles peuvent aussi être seulement inspirées, avec plus ou moins de talent, par leur peinture ${ }^{26}$. Avant l'invention

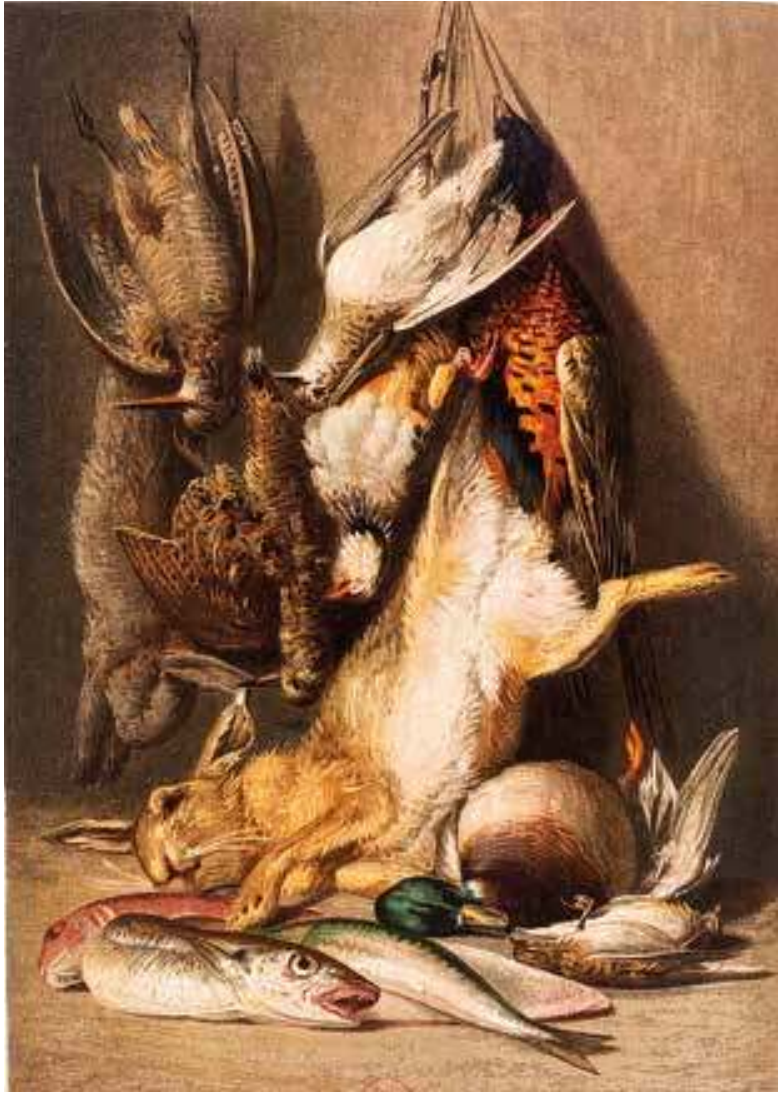

3. Eugène Ronjat (dessin), planche d'ouverture publiée dans Jules Gouffé, Le Livre de cuisine : la cuisine de ménage et la grande cuisine avec 25 planches imprimées en chromo-lithographie et 161 vignettes sur bois dessinées d'après nature par E. Ronjat, Paris, Hachette, 1867, n. p.. Paris, Bibliothèque nationale de France, département Sciences et techniques. et le développement de la photographie dans l'illustration des livres de recettes, les tableaux servaient déjà de faire-valoir.

Le Livre de cuisine de Jules Gouffé, ancien officier de bouche du Jockey Club de Paris, édité en $1867^{27}$ et ayant connu un grand succès, s'appuyait sur des illustrations de la main d'Eugène Ronjat, rendu célèbre pour sa copie du Radeau de la Méduse, qui lorgnait vers les grands modèles de la peinture classique et, notamment, la nature morte, comme en témoigne sa composition au lièvre et poisson au début de l'ouvrage (fig. 3).

La photographie, qui s'est peu à peu imposée comme moyen d'illustrer les livres de cuisine ${ }^{28}$, rendant obsolète tout autre moyen d'illustration jugé soudainement trop modeste, s'est progressivement transformée. D'une technique descriptive, visant à offrir aux lecteurs le résultat qu'il devait atteindre, jusque dans le dressage (les plats sont le plus souvent montrés sur des assiettes, prêts à être dégustés, et rarement dans les casseroles qui ont permis leur réalisation) qui accompagne la recette proprement dite ( "Sur chaque assiette, déposer une poitrine et une cuisse de pigeon. Accompagner de deux ravioles de betterave ${ }^{29}$ »), elle est devenue " artistique ». Les livres de cuisine exploitent désormais presque systématiquement la valeur ajoutée de l'art photographique qui s'exprime bien au-delà de l'illustration de la recette elle-même. De très nombreux 


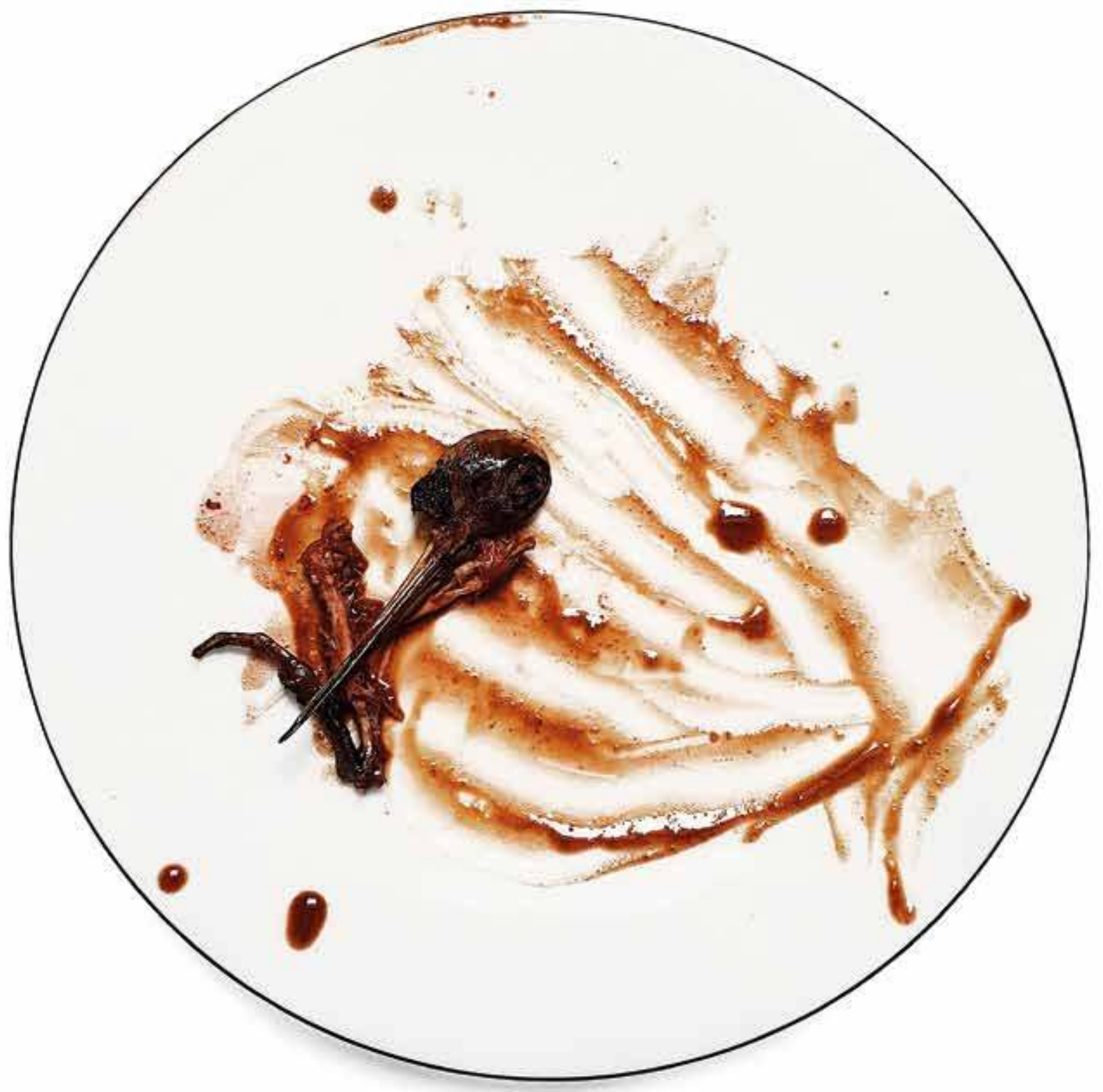


livres de ce type utilisent les photographies comme un accompagnement généralisé, où les différents genres de l'art de la photographie sont convoqués : le portrait (du chef, de sa brigade, de ses fournisseurs...), le paysage (le lieu où se trouve le restaurant, celui d'où viennent les produits travaillés...) $)^{30}$ et, naturellement, la nature morte, qui est le modèle indépassable pour l'immortalisation des plats.

Le dispositif retenu par Éric Poitevin pour photographier les plats réalisés par les Troisgros prend à la lettre, malgré son choix radical, cette tradition esthétique (par le motif imposé comme par le souci d'esthétisme et de raffinement, si important également dans les pratiques sociales de table où l'apparence vestimentaire compte) mais, en la poussant jusqu'à l'excès, en révèle les limites. Celles-ci touchent au sujet lui-même, en entraînant la décomposition du motif jusqu'à sa plus extrême limite (le crâne et un os d'une bécasse, comme dans l'illustration p. 135 [fig. 4], pour un plat justement appelé « Bécasse au crépuscule »), mais aussi les conventions morales attachées à ces pratiques de table. De la nature morte, Poitevin retient la mort des êtres de la nature, comme il l'avait fait dans un de ses livres précédents en ficelant des cadavres d'oiseaux par les pieds ${ }^{31}$, exposant la mort dans la nature, dans un geste (la ligature) qui n'est pas éloigné de celui du cuisinier apprêtant ses viandes. Des aliments disposés ( "dressés ", dit-on dans le langage culinaire) sur l'assiette, Poitevin ne montre presque plus rien et se contente de dévoiler ce rien qui est le résultat de la voracité des convives, accentuant ainsi ou révélant par là la charge de violence propre à ces pratiques alimentaires, notamment lorsqu'il s'agit de nourriture carnée. De nouvelles sensibilités contemporaines, soucieuses du bien-être animal, alertent désormais sur certaines attitudes, largement partagées dans le monde de la cuisine (la viande associée à la vitalité, l'énergie, la virilité...), ayant construit depuis des générations une " conscience alimentaire $^{32}$ ", qui deviennent soudainement dérangeantes ${ }^{33}$. Les gravures du lièvre cuit à la broche, piqué de lard fin, dans le livre de recettes de Jules Gouffé, pourraient aujourd'hui rebuter plus d'un cuisinier ou d'une cuisinière en herbe ${ }^{34}$, et les descriptions des opérations pour préparer les viandes risquent de ne plus passer aussi simplement qu'avant : "Au couteau, inciser le ventre du lièvre sur sa longueur. En extraire les viscères. Hacher finement le cœur et le foie ${ }^{35}$. " Les recettes de François Pierre dit de La Varenne, tirées de son Cuisinier françois de 1651, dont Lebensztejn reproduit des extraits en regard de son texte (Servez citron... est un livre de feuilletés), sont encore plus implacables : " Pour les mettre en ragoût, prenez les [pigeonneaux] sortant de dessous la mère, tuez-les, échaudez aussitôt ${ }^{36} \ldots$ "

En ne retenant que les " restes ", Poitevin donne à voir l'obscène du repas, son envers, ce qui doit rester tu mais qui pourtant demeure perceptible et que les bonnes manières tentent, tant bien que mal, d'exclure. Lebensztejn y insiste en montrant que toutes ces règles de civilité désignent, en sous-main, le dégoût du goût ${ }^{37}$ que le fait de se nourrir révèle : ne pas faire de bruit en mangeant, ne pas parler la bouche pleine, ne pas relâcher de vents (le Diogène françois de 1617, cité par Marie-France Lecherbonnier dans son Savoir-vivre de 1994, conseille aux femmes d'avoir un petit chien avec elles pour « servir de couverture aux exhalaisons du ventricule $\left.{ }^{38} »\right)$. Montrer des assiettes vidées, et non pas vides, fait revenir paradoxalement le corps que l'on souhaite si systématiquement, dans la culture occidentale au moins ${ }^{39}$, occulter : « La civilité d'Occident consiste pour une part, symbolique de son tout, à interposer quelque chose entre le corps

4. Éric Poitevin, photographie illustrant la recette "Bécasse au crépuscule", publiée dans Servez citron..., 2020, p. 135. et le corps $[\ldots]$. La bouche se clôt pour mastiquer, comme s'il s'agissait de couvrir d'un voile le mystère de l'animalité qu'on ne peut tuer en soi $^{40}$. " 


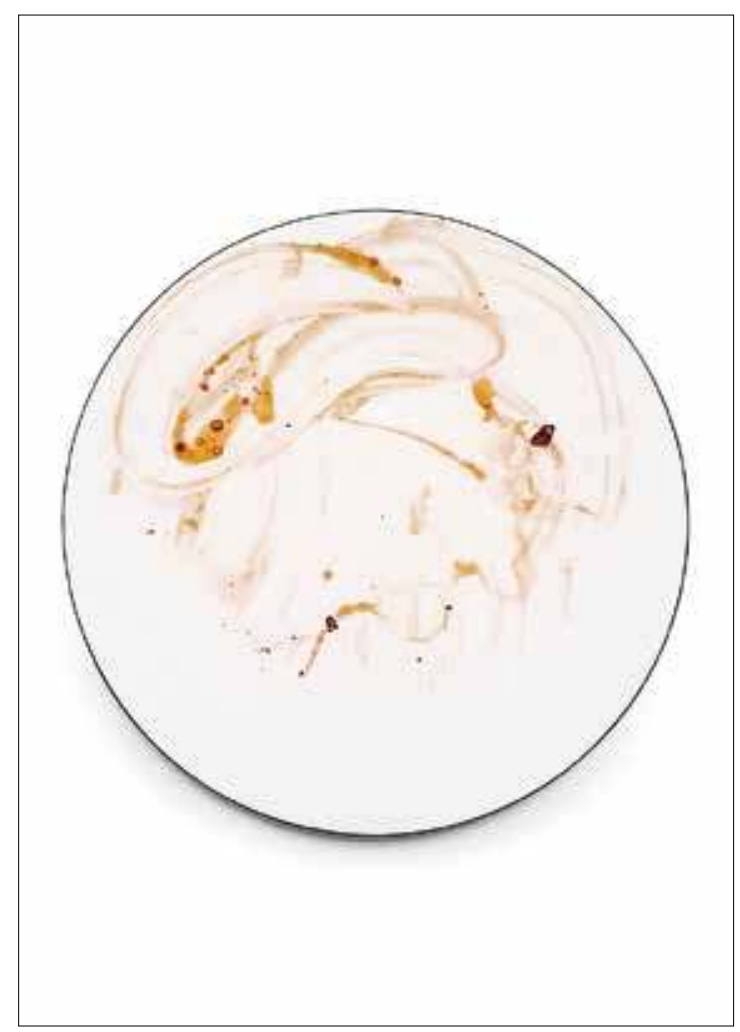

5. Éric Poitevin, photographie illustrant la recette "Chevreuil au gomasio ", publiée dans Servez citron..., 2020, p. 137.
Poitevin, en photographiant ce qui reste d'un repas, aussi bon et raffiné soit-il, révèle la part maudite de la nourriture et de son ingestion. L'assiette du pigeon aux baies de cassis, reproduite page 129, dévoile ainsi, par le bout d'os encore attaché à la chair, la manière dont la viande a été découpée pour être mangée et le rapport d'un corps dévorant à un corps mort que les photographies habituelles des livres de cuisine édulcorent. Il y a bien, dans les motifs retenus par la photographie, des qualités indéniablement picturales (les assiettes sont, de la Renaissance à Matisse, un support habituel de peintures et, dans un registre plus populaire, d'images. J'aimais beaucoup, enfant, boire ma soupe dans une assiette creuse décorée en son fond d'un paysage de campagne avec des personnages cheminant que je découvrais au fur et à mesure), des tracés colorés qui rappellent le pinceau. Barthes voyait, dans les plateaux des repas japonais, des palettes qui transformaient le convive en peintre qui, en piochant ici et là les aliments disposés, composait son œuvre ${ }^{41}$. Les recettes ("Orientalisme", "L'Ange qui passe ") en appellent aussi à cet imaginaire de la peinture que les photographies soulignent par endroits - l'aplat ( "L'escalope de saumon à l'oseille », p. 105), le pointillisme ( "Orientalisme ", p. 141 ou "Saké-Sakura ", p. 150), l'écoulement ("Cosa croccante", p. 81), l'expressionnisme abstrait ( " Pigeons aux baies de cassis ", p. 128) -, et qu'elles rejouent sur différents plans : le jeu entre le relief et le plan (on pense au fameux Flatbed Picture Plane avec lequel Leo Steinberg avait décrit le redressement vertical de l'espace horizontal de la représentation classique ${ }^{42}$ ), la surface et la profondeur, l'abstraction et la figuration (quelques images doubles s'y trouvent, comme ces visages dans l'assiette du "Chevreuil au gomasio », p. 137 [fig. 5], ou dans celle du « Dim Sum au tamarin et au miel ", p. 144).

En lisant, regardant, savourant par l'imagination ou en mettant la main à la pâte (la "Ronde d'écrevisses et de framboises ", pour ma part), le lecteur et, il faut l'espérer, notamment celui si peu habitué à lire dans une telle revue ce type de compte rendu, se voit envahi par ce que Flaubert appelait la "verve " : " Être en verve c'est avoir envie de se mettre à table, sans céder tout de suite à cette envie. " En tournant les pages de ce livre, dans l'ordre ou le désordre, le lecteur est entraîné dans cette " danse préalable ", cet « emportement léger qui fait tourner, sans les toucher, autour des idées et des choses, les présentant à l'imagination dans la multiplicité de leurs jouissances futures ${ }^{43}$ ". 


\section{Guillaume Cassegrain}

Guillaume Cassegrain est professeur d'histoire de l'art moderne à l'université Grenoble Alpes. Amateur de cuisine italienne, il a travaillé sur Michel-Ange ou sur Tintoret, celui que l'on surnommait le " grain de poivre " et qui avait su épicer la peinture vénitienne de son temps.

\section{NOTES}

1. Sur les liens entre virus, alimentation et économie, voir Andreas Malm, La Chauve-souris et le capital. Stratégie pour l'urgence chronique, Étienne Dobenesque (trad. fr.), Paris, La fabrique éditions, 2020. Quelques chefs étoilés se sont emparés de ces problèmes économiques et écologiques que l'industrie alimentaire et le monde de la gastronomie, destinés à une clientèle privilégiée, ne souhaitaient pas voir apparaître. Voir, par exemple, Olivier Roellinger, Pour une révolution délicieuse, Paris, Fayard, 2019.

2. Michel de Montaigne, "De la vanité des paroles", Essais (1580-1588), livre I, chapitre LI.

3. Cécile Montier, La Bonne Cuisine de tante Louise, Paris, 1930 ; Tante Marie, La Véritable Cuisine de famille, Paris, 1937 ; Les Bonnes Recettes de tante Cécile, Paris, 1978. Les versions masculines des oncles existent naturellement avec Les Bonnes Recettes de I'oncle Jean, Paris, 1934 ; Benoît Mascarelli, La Bonne Cuisine de I'oncle Benoît, Paris, 1946.

4. Les copyrights à la fin du livre ne mentionnent que les éditions Macula pour " la présente édition » et Éric Poitevin pour les photographies. Les recettes ne semblent être protégées que par le détour de l'éditeur.

5. Par exemple, par Gina Beavers qui exploite l'imagerie véhiculée par les photographies de plats échangés sur les réseaux sociaux tout en exaltant la dimension sexuelle de la nourriture, mêlant ainsi "foodporn » et « porn ». Voir "The Life I Deserve ", MoMA PS1, New York, 31 mars - 2 septembre 2019.

6. Sade, La Nouvelle Justine ou les malheurs de la vertu, VII, 80, cité par Béatrice Bomel-Rainelli dans "Sade ou l'alimentation générale ", dans Dix-Huitième siècle, $\mathrm{n}^{\circ}$ 15, 1983, p. 203. Voir Jean-Claude Lebensztejn, Kafka, Sade, Lautréamont : rêve déchiré, Dijon, Les Presses du réel, 2017, p. 51-85. Dans un autre genre, pas du meilleur goût, ce " bon mot» de Paul Bocuse qui pointait du doigt, à sa manière, les liens entre sexualité et nourriture: "Il y a beaucoup de points communs entre la cuisine et le sexe. On consomme une union, on dévore des yeux, on a faim de l'autre. L'homme a toujours besoin de manger et de se reproduire, et dans les deux cas, il faut passer à la casserole. »

7. Sur cette "manducation sensuelle " analysée par Jean-Pierre Richard, L'Univers imaginaire de Mallarmé, Paris, Éditions du Seuil, 1961, voir Jacques Dupont, "Jean-Pierre Richard ou la critique comme métier de bouche » dans un numéro spécial de la revue Critique, "La gastronomie », nº $685-686,2004$, p. 507. On pourra aussi, sur ce rapport entre mots et mets, se reporter aux analyses de Louis Marin sur la « chair à pâté » dans le conte "Le Maître chat ou le Chat botté ", dans La Parole mangée et autres essais théologico-politiques, Paris, Klincksieck, 1986, p. 161-166. Voir aussi Michel Jeanneret, Des Mets et des mots. Banquets et propos de table à la Renaissance, Genève, Droz, 1989.

8. Roland Barthes, « Lecture de Brillat-Savarin », préface de Jean Anthelme Brillat-Savarin, Physiologie du goût, Paris, Hermann, 1975, repris dans CEuvres complètes, t. IV, Paris, Éditions du Seuil, 2002, p. 810.

9. Alexander Cockburn, "Gastro-porn", dans The New York Review of Books, 8 décembre 1977 [en ligne, URL : https://www.nybooks.com/articles/1977/12/08/ gastro-porn/].

10. Michel Troisgros, Michel Troisgros et l'Italie, Grenoble, Glénat, 2009, p. 64.

11. La recette de Coelius Apicius vient du De opsoniis et condimentis, sive arte coquinaria, livre 10 dans l'édition hollandaise de Martin Lister de 1709. Il s'agit de boulettes de foie de porc travaillées avec de la sauce de poisson fermentée, de la cervelle cuite. Voir Carl Friedrich von Rumohr, L'Esprit de l'art culinaire, Andreas Beyer (préface), Marie-Ange Maillet (trad. fr.), Paris, Klincksieck, 2016, p. 20-21. Rumohr critique les livres de cuisine allemands qui ne font que singer les livres français et reprendre leur terminologie « inutile et barbare », p. 24.

12. Voir, pour quelques noms de ces photographes spécialistes, Françoise Hache-Bissette, "L'évolution du livre de cuisine : du livre pratique au beau-livre ", dans Le Temps des médias, $\mathrm{n}^{\circ}$ 24, 2015, p. 106.

13. Jean-Claude Lebensztejn, "Restes de table», dans Servez citron..., 2020, p. 36.

14. Madame la comtesse de Boissieu, Le Vrai Manuel du savoir-vivre. Conseils sur la politesse et les usages du monde, Paris, Gauguet et compagnie, 1877, p. 17.

15. Dans la classification française de l'édition, les livres de cuisine relèvent de la section "Livres pratiques", voir Hache-Bissette, 2015, cité n. 12, p. 97.

16. Servez citron..., 2020, p. 197 (à propos du rouget à la moelle).

17. Servez citron..., 2020, p. 211 (à propos du carré d'agneau brûlé). Le film en question désigne naturellement la matière fine plastique que l'on étend pour préserver un aliment. On peut aussi « filmer » le plat mais c'est une autre affaire, qui témoigne également de ce développement des outils médiatiques dans le rapport à la cuisine.

18. Algirdas Julien Greimas, "La soupe au pistou ou la construction d'un objet de valeur ", dans Du sens II. Essais sémiotiques, Paris, Éditions du Seuil, 1983, p. 160.

19. Voir, parmi l'abondante bibliographie sur le sujet, Domenico Bertoloni Meli, Visualizing Disease. The Art and History of Pathological Illustrations, Chicago, The University of Chicago Press, 2018. Bertolini Meli cite notamment le cas de Fabricius Hildanus qui s'appuie sur les gravures dans ses Observationes et curationes chirurgicae (1646) car les problèmes posés au chirurgien, dit-il, " restent obscurs par la description et se comprennent mieux par les images», p. 28. 
20. Voir The Technical Image: A History of Styles in Scientific Imagery, Horst Bredekamp, Vera Dunkel and Birgit Schneider (éd.), Chicago, The University of Chicago Press, 2015.

21. Voir Deborah L. Krohn, "Le livre de cuisine de la reine : un exemplaire de l'Opera de Scappi dans la collection de Catherine de Médicis ", dans Pascal Brioist et Florent Quellier (dir.), La Table à la Renaissance. Le mythe italien, Rennes / Tours, Presses universitaires de Rennes / Presses universitaires François Rabelais, 2018, p. 151-163. Ce livre fait partie d'une collection, "Tables des hommes», consacrée à I'alimentation.

22. Paris, Plon, 1963, préface de Jean Cocteau.

23. Sur ce point, voir Hache-Bissette, 2015, cité n. 12, p. 104.

24. Greimas, 1983, cité n. 18, p. 160.

25. Les exemples sont innombrables. Nous citerons $L a$ Cuisine selon Renoir, Jean-Michel Charbonnier, photographies de Jean-Bernard Naudin, Paris, Le Chêne, réédité en 2009, car il est préfacé par Pierre Troisgros, le chef renommé venant ajouter une valeur à celle du célèbre artiste. Dans cette préface, Pierre Troisgros se félicite d'ailleurs de ces rapprochements opérés par les éditeurs : «Beaucoup de cuisiniers sont proches de l'art pictural et se réjouissent toujours des comparaisons avec l'art culinaire. » Une collection des éditions du Chêne a permis de décliner ces confrontations avantageuses. La Cuisine selon Cézanne est elle, par exemple, préfacée par Alain Ducasse. Même les peintres qui ne semblent pas les plus à même de cuisiner et de recevoir, avec toutes les manières attendues, leurs hôtes ont eu droit à ce type d'ouvrages. Voir Robyn Lea, Dinner with Jackson Pollock Recipes, Art and Nature, New York, Assouline, 2015, livre réalisé à partir des recettes notées par Lee Krasner et Jackson Pollock. Willem de Kooning rapporte une anecdote que lui avait racontée Franz Kline sur Jackson Pollock et sur ses « manières de table » : «Au milieu du repas, voyant que le verre de Franz est vide, Pollock dit : "Franz, je te sers du vin." Il se met alors à remplir le verre et, complètement fasciné par le filet de vin qui s'écoule de la bouteille, il vide toute la bouteille. Du vin, il y en avait partout, dans les assiettes, sur la table, partout [...]. Comme un gamin, il avait trouvé l'idée grandiose - tout ce vin qui coulait partout. Après quoi, il prit la nappe par les quatre coins et l'a étalée par terre ", Willem de Kooning, Écrits et propos, Christian Bounay, Marie-Dominique Nobécourt, Dick Van Leeuwen (trad. fr.), Paris, École nationale supérieure des Beaux-Arts, 1992, p. 127-128.

26. Voir Yves Pinard et Séverine Quoniam, Cuisine et peinture au Louvre, Grenoble, Glénat, 2004, par exemple.

27. Jules Gouffé, Le Livre de cuisine. La cuisine de ménage et la grande cuisine..., Paris, Hachette, 1867.

28. Hache-Bissette, 2015, cité n. 12, p. 106.

29. Servez citron..., 2020, p. 213 (à propos du « Pigeon aux baies de cassis »).

30. Voir Jean Sulpice. Le chef, l'Auberge et le lac, Grenoble, Glénat, 2020. Modèle représentatif de ce genre, avec une édition soignée qui fait du livre luimême un travail artistique, des photographies " d'art » de Thomas Dhellemmes et un texte de Luc Le Vaillant qui joue sur le registre littéraire.
31. Éric Poitevin, Jean-Christophe Bailly, Le Puits des oiseaux. Nature morte, Paris, Éditions du Seuil, 2016. Dans la recette de la bécasse, les Troisgros disent qu'il n'est pas nécessaire de « brider » les cuisses de l'oiseau (p. 223).

32. Roland Barthes, "Pour une psycho-sociologie de l'alimentation contemporaine ", dans Annales, 16 e année, n 5, octobre 1961, repris dans OEuvres complètes, t. I, Paris, Éditions du Seuil, 2002, p. 1113.

33. Une très abondante littérature culinaire végane s'est développée pour répondre à ces nouvelles attentes. Voir, par exemple, Jonathan Safran Foer, Eating Animals, New York, Little, Brown and Company, 2009.

34. Gouffé, 1867, cité n. 27, p. 202.

35. Servez citron..., 2020, p. 217.

36. Servez citron..., 2020, p. 47.

37. Voir Jacques Derrida, "Economimesis ", Mimesis des articulations, collectif, Paris, Aubier-Flammarion, 1975, p. 57-93.

38. Servez citron..., 2020, p. 52.

39. Jean-Claude Lebensztejn rapporte que cette présence du corps n'est pas un problème ailleurs : les Japonais « sucent leurs nouilles bruyamment » (Servez citron..., 2020, p. 36).

40. Servez citron..., 2020, p. 36.

41. « La peinture n'était au fond qu'une palette (une surface de travail), dont vous allez jouer au fur et à mesure que vous mangerez ", L'Empire des signes, repris dans OEuvres complètes, t. III, Paris, Éditions du Seuil, 2002, p. 358.

42. Leo Steinberg, Other Criteria, Confrontations with Twentieth-Century Art, Chicago, The University of Chicago Press, 1972. Dans une interview, Steinberg raconte les difficiles adaptations aux mœurs alimentaires américaines pour les expatriés : "We were never friends [Steinberg parle des artistes abstraits de l'époque qu'il pouvait rencontrer]. We met in various ways. Once, Alfred Barr invited a number of artists and people in the art world to a party to meet some visiting Russians. This was long ago, of course, in the Soviet days, and the party was held at the home of a real upper class Park Avenue Wasp. A few canapes were served and of course drinks, and the Russians were waiting for the food, and there was no food; that was it. The Russian idea of hospitality was not that, you see, and this was very Wasp. The Russians were duly appalled. And so was Rothko, who was there. Everybody was properly dressed; I certainly wore my tie, but Rothko came in an open shirt, with his hairy chest showing. Then he turned to a few people - I was sitting near him - and said, "Let's go get something to eat." We all lit out and went to a delicatessen. I think that may have been the first time I talked to Rothko. Then I remember hearing about the piece he had been commissioned to do for the Four Seasons restaurant, the Philip Johnson restaurant ", dans The Gestural Trace. Leo Steinberg interviewed by R. C. Smith (Getty Research Institute, Art History Oral Documentation Project, 1998), Los Angeles, The John Paul Getty Trust, 2001, p. 58.

43. Jean-Pierre Richard, Stendhal et Flaubert. Littérature et sensation, Paris, Éditions du Seuil, 1970, p. 138. 